\title{
LA METODOLOGÍA DE LAS CIENCIAS SEGÚN BOECIO: SU RECEPCIÓN EN LAS OBRAS Y TRADUCCIONES DE DOMINGO GUNDISALVO'
}

\author{
Alexander Fidora \\ Johann Wolfgang Goethe-Universität \\ Frankfurt am Main
}

\section{RESUMEN}

Los estudiosos que se han ocupado durante el último siglo de la transmisión del saber en la obra de Gundisalvo, han prestado mucha -por no decir exclusiva- atención a su recepción e integración de elementos arábigo-juđíos. Sin embargo, para entender adecuadamente este proceso de integración parece imprescindible analizar también la recepción de la tradición latino-cristiana en la obra del arcediano, ya que ésta condiciona dicho proceso. Partiendo de la recepción de la metodología de las ciencias según Boecio por Gundisalvo, el presente estudio pretende demostrar cómo los problemas que Gundisalvo encontró en su propia tradición influyen en su recepción y traducción de la filosofía arábigo-judía.

Palabras clave: Boecio, Gundisalvo, metodología de las ciencias, noética, transmisión del saber.

\begin{abstract}
Scholars during the last century dealing with the transmission of knowledge in the work of Gundissalinus have been paying much -if not exclusive-attention to his reception and integration of Arabo-Jewish elements. However, it seems indispensable for a suitable understanding of this process of integration to take into account also the archdeacon's reception of the Latin-Christian tradition, for it conditions the aforesaid process. Starting from Gundissalinus' reception of Boethius' methodology of the sciences, the present article pretends to show how the problems Gundissalinus encountered in his own tradition affect his reception and his translation of the Arabo-Jewish philosophy.
\end{abstract}

Key words: Boethius, Gundissalinus, methodology of the sciences, noetics, transmission of knowledge.

\section{JUSTIFICACIÓN DEL TEMA}

El arcediano de Cuéllar, Domingo Gundisalvo (*ca. 1110, †ca. 1181), puede ser considerado con pleno derecho como el representante más importante de la llamada Escuela de Traductores de 
Toledo - uno de los centros más destacados para la transmisión del saber en la Edad Media- ${ }^{2}$ en donde bajo el mecenazgo del arzobispo Ramón de Toledo (†1152) fueron traducidas varias obras de autores arábigo-judíos al latín, así como más tarde también el Corpus aristotelicum arabum. Esta importancia sistemática radica en que Domingo Gundisalvo no tan sólo tradujo junto con su colaborador Avendauth (Ibn Dawud) -cuya identidad no ha podido ser determinada con seguridad hasta ahora- unas veinte obras del árabe al latín de filósofos tan notables como al-Farabi, Avicena y alGazali, sino que además es el autor de los siguientes cinco tratados: el De processione mundi, el Tractatus de anima, el De immortalitate animae -probablemente plagiado por Guillermo de Auvernia-, el opúsculo De unitate -atribuido durante siglos a Boecio-y, para acabar, su obra más influyente con el título De divisione philosophiae. ${ }^{3}$

En los más de cien años transcurridos desde la publicación de estos tratados, promocionada sobre todo por Clemens Baeumker y sus Beiträge zur Geschichte der Philosophie des Mittelalters, los estudiosos de estos tratados han prestado una atención especial a la integración de fuentes arábigo-judías por Domingo Gundisalvo, hasta el punto que parece que Gundisalvo haya recibido más atención por parte de los arabistas -de los cuales aquí solamente nombraré al padre Manuel Alonso y a Marie-Thérèse d'Alverny- que no de los historiadores de la filosofía medieval. Así que, bien que por los aparatos críticos de las respectivas ediciones de sus obras se conozcan las fuentes latino-cristianas de Domingo Gundisalvo, faltan estudios detenidos sobre la interpretación y utilización concretas de las mismas en los tratados del arcediano.

Claro está, que lo «nuevo» o «desconocido» en las obras de Gundisalvo está en la transmisión e integración de elementos arábigo-judíos -y es esto lo que primero, y con razón, llamó la atención a sus estudiosos-; pero es precisamente para entender mejor esta integración de lo «desconocido», es decir la tradición arábigo-judía, que hay que preguntarse cómo entendió Gundisalvo lo «conocido», es decir su propia tradición latino-cristiana. Sólo de esta manera se entenderá plenamente lo

2 Véase para la Escuela de Traductores de Toledo, entre otros, Brasa Díez, Mariano, «Métodos y cuestiones filosóficas en la Escuela de Traductores de Toledo», en Revista Española de Filosofía Medieval, 3 (1996), pp. 35-49. La existencia real de tal Escuela ha sido puesta en duda en varias ocasiones, en último lugar por parte de Serafín Vegas González en su muy bien documentado monográfico, La Escuela de Traductores de Toledo en la Historia del Pensamiento, Toledo, Ayuntamiento de Toledo, 1997 (véase la reseña correspondiente de Lomba, Joaquín en Revista Española de Filosofía Medieval, 6 [1999], pp. 295-296). Sin embargo, para nuestro propósito no es decisiva la existencia física de tal Escuela; se emplea aquí el concepto de Escuela en un sentido amplio, así como se suele hacer también en otros casos, por ejemplo en el de la Escuela de Chartres.

3 Las obras de Domingo Gundisalvo han sido publicadas en los siguientes lugares: De processione mundi, ed. Georg Bülow, en Beiträge zur Geschichte der Philosophie des Mittelalters (= BGPhMa), XXIV, 3, Münster, 1925, pp. 156, y antes ed. Marcelino Menéndez y Pelayo, en Historia de los heterodoxos españoles, vol. 1, Madrid, 1880, pp. 691-711, trad. española en la memoria de la licenciatura salamantina de Noboru Kinoshita; Tractatus de anima, ed. Joseph Thomas Muckle, en Mediaeval Studies, 2 (1940), pp. 23-103; De immortalitate animae, ed. Georg Bülow, en BGPhMa, II, 3, Münster, 1897, pp. 1-38, trad. española en Kinoshita, Noboru, El pensamiento filosófico de Domingo Gundisalvo, Salamanca, Universidad Pontificia, 1988, pp. 129-149; De unitate, ed. Manuel Alonso, en Pensamiento, 12 (1956) y 13 (1957), pp. 65-78, pp. 179-202, pp. 431-472 y pp. 159-202, antes ed. Paul Correns, en BGPhMa, I, 1, Münster, 1891, pp. 1-11, trad. española en Kinoshita, Noboru, op. cit., pp. 123-128; De divisione philosophiae, ed. Ludwig Baur, en BGPhMa IV, 2-3, Münster, 1903, pp. 1-141, trad. parcial al inglés en Grant. Edward (ed.), A Source Book in Medieval Science, Cambridge (Mass.), 1974, pp. 59-76; - Para una valoración más detallada de la vida y las obras de Domingo Gundisalvo y el status quaestionis de la investigación actual véase mi artículo s.v. Dominicus Gundissalinus en BiographischBibliographisches Kirchenlexikon, XVII (2000). 
que supone la transmisión del pensamiento arábigo-judío, mediador del aristotelismo, que desde Toledo, en pocos años, conquistaría el mundo occidental entero. Hace falta, por lo tanto, preguntarse cuáles eran las condiciones y los problemas dentro de la propia tradición latino-cristiana que permitirían a Gundisalvo recoger y asimilar elementos de una filosofía hasta entonces desconocida por los centros intelectuales más prominentes de occidente.

A continuación, me propongo estudiar tan sólo un aspecto de este background intelectual de la integración de la filosofía arábigo-judía: El uso que Gundisalvo hace de la metodología de las ciencias desarrollada por Boecio. Parece que éste último junto con San Isidoro de Sevilla ${ }^{4}$ haya sido la principal fuente latino-cristiana del arcediano: En todas sus obras se encuentran numerosas referencias al De consolatione philosophiae, al De institutione arithmetica, al De institutione musica, al De differentiis topicis, al In Ciceronis topica y, por supuesto, al De trinitate. ${ }^{5}$ Ya tan sólo la gran abundancia de citas hace sospechar que Gundisalvo tenía acceso a manuscritos boecianos, lo que se ve confirmado por el ms. 10109 de la Biblioteca Nacional de Madrid proveniente del Toledo del siglo XII y que contiene la Consolatio. ${ }^{6}$

\section{LA METODOLOGÍA DE LAS CIENCIAS SEGÚN BOECIO Y SUS COMENTARISTAS}

El locus classicus de la metodología de las ciencias de Boecio es su tratado De trinitate ${ }^{7}$. En éste, antes de entrar en la discusión del tema propuesto, a saber la trinidad, Boecio discute ampliamente la división de las ciencias en scientia naturalis, mathematica y scientia divina. Primero, siguiendo a Aristóteles Met. VI y su división de las ciencias, expone que a cada una de ellas le corresponde un objeto distinto: Así la ciencia natural trata de los objetos que se encuentran in motu inabstracta, la matemática de los objetos que se encuentran sine motu inabstracta y la ciencia divina de éstos que están sine motu abstracta: ${ }^{8}$

4 Para un estudio de la influencia de San Isidoro en Domingo Gundisalvo véase mi artículo, «La recepción de San Isidoro por Domingo Gundisalvo: Astronomía, astrología y medicina», a aparecer en Estudios Eclesiásticos, 76 (2001), donde se intenta demostrar cómo San Isidoro provee a nuestro arcediano con las categorías hermenéuticas fundamentales, tanto a nivel formal como material, para integrar las nuevas ciencias arábigas en su ordo scientiarum.

$5 \mathrm{El}$ padre Manuel Alonso ha proporcionado una relación completa de las citas de Boecio en las obras de Gundisalvo, «La influencia de Severino Boecio en las obras y traducciones de Gundisalvo», en íd., Temas filosóficos medievales (Ibn Dawudy Gundisalvo), Comillas, Universidad Pontificia, 1959, pp. 369-396. Lamentablemente se limita a enumerar las citas correspondientes sin intentar dar una interpretación de las mismas.

6 Véase para este manuscrito Rubio Fernández, Lisardo, Catálogo de los manuscritos clásicos latinos existentes en España, Madrid, 1984. Sin embargo, no me ha sido posible hasta ahora localizar otros manuscritos boecianos que pudieran estar al alcance de Domingo Gundisalvo. Agradezco el apoyo en esta búsqueda por parte del director de la Biblioteca Capitular de Toledo Ramón Gonzálvez.

7 Desgraciadamente no existe edición crítica del De trinitate ni de los otros Opuscula sacra. La edición más provechosa sigue siendo la de Peiper, Rudolf, Anicii Manlii Severini Boetii Philosophiae consolationis libri quinque accedunt eilusdem atque incertorum Opuscula sacra, Lipsiae, Teubner, 1871. Esta edición ha servido como base a todas las ediciones modernas aventajándolas en que ofrece un pequeño aparato crítico que reúne algunas lecciones de diferentes manuscritos (principalmente del área alemana). De las ediciones modernas nos referimos a Stewart, H. F., Rand, E. K. y Tester, S. J., Boethius: Tractates. The Consolation of Philosophy; Cambridge (Ma.), Loeb, 1918, reimpr. en varias ocasiones, la más reciente 1997, que ofrece también una traducción inglesa. Se citará según ambas ediciones; la primera como Peip. y la segunda como Stew. 
Nam cum tres sint speculativae partes, naturalis, in motu inabstracta ' $\alpha v v \pi \varepsilon \xi \alpha$ i $\rho \varepsilon \tau 0 \varsigma$ (considerat enim corporum formas cum materia, quae a corporibus actu separari non possunt [...]), mathematica, sine motu inabstracta (haec enim formas corporum speculatur sine materia ac per hoc sine motu, quae formae cum in materia sint, ab his separari non possunt), theologica, sine motu abstracta atque separabilis (nam dei substantia et materia et motu caret) $[\ldots]^{9}$

En segundo lugar, Boecio distingue las ciencias según sus métodos:

In naturalibus igitur rationabiliter, in mathematicis disciplinaliter, in divinis intellectualiter versari oportebit. ${ }^{10}$

He aquí pues en una sola frase la metodología de las ciencias que tanto intrigó a los comentaristas de Boecio del siglo XII, siendo el método matemático y teológico el que más les llamó la atención. ${ }^{11}$ El primero y más destacado entre ellos, el obispo de Poitiers Gilberto ( ${ }^{*}$ ca. 1080, †1154), comenta el pasaje boeciano de la siguiente manera:

Ac per hoc 'in naturalibus' quae sicut sunt percipi debent, scilicet concreta et inabstracta, 'oportebit' philosophum 'versari rationaliter' [...] 'In mathematicis' vero ubi inabstracta aliter quam sint i.e. abstractim attenduntur, oportebit eum versari 'disciplinaliter' [...] 'In divinis' quoque quae non modo disciplina verum etiam re ipsa abstracta sunt 'intellectualiter' versari oportebit $[\ldots]^{12}$

La explicación que Gilberto da aquí de la metodología de las ciencias es, de hecho, una reaplicación de uno de los criterios con los cuales Boecio distingue los objetos de las diferentes ciencias, a saber la abstracción, a sus respectivos métodos. Con esto la metodología de las ciencias parece reducirse a la distinción de sus objetos. ${ }^{13}$

Mucho más diferenciada a este respecto es la propuesta de Thierry de Chartres (†1148/1155), canciller de la Escuela de Chartres, en su comentario al De trinitate:

8 En la conferencia internacional «Scientia und Disciplina im 12. und 13. Jahrhundert: Wissenstheorie und Wissenschaftspraxis im Wandel», 3 al 4 de diciembre de 1999, Frankfurt am Main, organizada por Matthias LutzBachmann, Alexander Fidora y Andreas Niederberger, he tenido ocasión de dedicarme precisamente a la recepción de esta clasificación de las ciencias según sus objetos por Domingo Gundisalvo. Mi contribución aparecerá bajo el título «Die Rezeption der boethianischen Wissenschaftseinteilung bei Dominicus Gundissalinus» en las actas del congreso que serán publicadas el año 2001 en el Akademie Verlag de Berlín.

9 Boecio, De trinitate, ed. Peip. p. 152, 11. 4-14 / Stew. p. 8, 11. 5-14.

10 Ibíd., ed. Peip. p. 152, 1l. 15-18/ Stew. p. 8, 11. 16-18.

11 Para una discusión del significado de la clasificación de las ciencias y de sus respectivos métodos según Boecio véase Neumann. Siegfried, Gegenstand und Methode der theoretischen Wissenschaften nach Thomas von Aquin aufgrund der 'Expositio super librum Boethii De trinitate', en BGPhMa, XLI, 2, Münster, 1965, pp. 36-57.

12 Gilberto de Poitiers, The Commentaries on Boethius by Gilbert of Poitiers, ed. Nikolaus M. Häring, Toronto, Pontificial Institute, 1966, pp. 86-87, nos. 38, 39 y 41.

13 Para más detalles sobre Gilberto y su comentario a los pasajes del De trinitate aquí presentados véase Jacobi, Klaus, «Natürliches Sprechen - Theoriesprache - Theologische Rede. Die Wissenschaftslehre des Gilbert von Poitiers (ca. 1085-1154)», en Zeitschrift für philosophische Forschung, 49 (1995), pp. $511-528$. 
Et sciendum quod diversis animae viribus et comprehensionibus in physica, mathematica, theologia utendum est ad comprehendendum universitatem ut subiecta est his tribus speculativae partibus. Nam in theologia utendum est intellectibilitate sive intelligentia: in mathematica vero intellectu qui est disciplina: in physica ratione sensu et imaginatione quae circa materiam comprehendunt quicquid comprehendunt. ${ }^{14}$

Para Thierry, la metodología de las ciencias está relacionada con las facultades del alma, con las «vires animae» (y no con los objetos), con lo que surge el problema siguiente: Mientras que el método de la física («rationabiliter») y el de la ciencia divina («intellectualiter») corresponden a una facultad del alma, a la razón y al intelecto, la matemática como ciencia que opera «disciplinaliter» no parece referirse a las capacidades del alma, sino al proceso de aprendizaje. Thierry intenta solucionar esta dificultad asignando a la matemática la facultad intelectual, es decir el intelecto («intellectu qui est disciplina»), mientras que determina como facultad de la ciencia divina lo que llama con un neologismo la «intellectibilitas» ${ }^{15}$ y que identifica a su vez con «intelligentia». ${ }^{16}$ De esto resulta una clara correspondencia entre las facultades del alma y los métodods de las ciencias: la física opera «rationabiliter» mediante la «ratio», la matemática «disciplinaliter» mediante el «intellectus» y la ciencia divina «intellectualiter» mediante la «intellectibilitas» o la «intelligentia». La única imperfección que resta en esta correspondencia es que el adverbio «intellectualiter» que según Boecio caracteriza el método de la ciencia divina se deriva morfológicamente de «intellectus» con lo que el intelecto se prestaría más bien para describir la ciencia divina que no la matemática.

Esta imperfección, aparentemente anodina, se volverá virulenta en el comentario al De trinitate de uno de los discípulos de Thierry, el arcediano Clarembaldo de Arras ( $† 1187)$ :

'Vel rationabiliter in naturalibus versari' oportet i.e. rationabiles causas inquirendo cur hoc tantum sit vel tale [...] 'In mathematicis vero disciplinaliter' i.e. abstractive et intellectibiliter philosopho versandum est [...] 'In divinis' autem 'intellectualiter' hoc es intellectibiliter theologum 'versari oportebit' $[\ldots]^{17}$

Clarembaldo sigue a su maestro en lo que se refiere a la explicación del método de la ciencia divina, «intellectualiter», mediante el nuevo concepto de «intellectibilitas» que aquí se encuentra

14 Thierry de Chartres, Commentaries on Boethius by Thierry of Chartres and His School, ed. Nikolaus M. Häring, Toronto, Pontificial Institute, 1971, p. 164, no. 30.

15 En cuanto al substantivo «intellectibilitas» se trata de un neologismo propio de la Escuela de Chartres. Véase Hamesse, Jacqueline, «Un nouveau glossaire des néologismes du latin philosophique», en íd. (ed.), Aux origines du lexique philosophique européen. L'influence de la 'latinitas', Louvain. Fédération Internationale des Instituts d'Études Médiévales, 1997, pp. 237-254, aquí pp. 249-251. Boecio conoce tan sólo el substantivo «intellectibilia» y el correspondiente adjetivo «intellectibilis» como lo demuestra el siguiente pasaje de su comentario a la Isagoge de Profírio: «Intellectibile, quod unum atque idem per se in propria semper divinitate consistens, nulli unquam sensibus, sed sola tantum mente intellectuque capitur; secunda vero est pars intellegibilis quae primam intellectibilem cogitatone atque intelligentia comprehendit [...]» (In Isagoge Porphyrii, ed. Samuel Brandt, Lipsiae, Teubner, 1906, pp. 8-9)

16 Para un buen análisis de la relación entre «intellectus», «intellectibilitas» y «intelligentia» en la obra de Thierry véase Dronke, Peter, «Thierry of Chartres», en íd. (ed.), A History of Tivelfth-Century Western Philosophy, Cambridge, University Press, 1988, pp. 358-385, y esp. pp. 365-367.

17 Clarembaldo de Arras, Life and Works of Clarembald of Arras. A Twelfth-Century Master of the School of Chartres, ed. Nikolaus M. Häring, Toronto, Pontificial Institute, 1965, pp. 113-114, nos. 17-19. 
bajo la forma «intellectibiliter». Sin embargo, el problema que surge a continuación es que el método «intellectibilter» es según Clarembaldo el mismo que también define la matemática. Tanto la ciencia divina como la matemática operan «intellectibilter». ${ }^{18}$ Con esto el intento de clarificación y sistematización de la metodología boeciana por parte de Thierry y su discípulo Clarembaldo resulta en una nivelación de la misma en la cual los diferentes métodos de las ciencias se confunden. Como se ha visto, la metodología de las ciencias de Boecio pone a sus comentaristas del siglo XII ante un problema que éstos no resuelven satisfactoriamente.

\section{CÓMO GUNDISALVO «CORRIGE» A BOECIO}

Domingo Gundisalvo expone la metodología de las ciencias de Boecio en una de sus obras tardanas, el De processione mundi ${ }^{19}$, su cosmología. En ella, después de citar al famoso verso 1, 20 de la Epístola de San Pablo a los Romanos, según el cual hay que ascender a partir de los seres creados y visibles a los invisibles, a Dios en última instancia, ${ }^{20}$ pone en paralelo con esta ascención la metodología de Boecio que cita de la siguiente manera:

Unde dicitur, quod in naturalibus rationaliter, in mathematicis disciplinaliter, in theologicis intelligentialiter versari oportet. ${ }^{21}$

Lo que podría parecer prima facie una cita literal de Boecio -y así lo señalan los editores del De processione mundi y también el padre Manuel Alonso- es en realidad mucho más que esto: En este pasaje Gundisalvo no tan sólo cita a Boecio, sino que lo «corrige» cambiando el «intellectualiter» que caracteriza la ciencia divina por «intelligentialiter». ${ }^{22}$

Después de está pequeña «corrección» Gundisalvo puede seguir parafraseando Boecio, esta vez la Consolatio V, pr. 4, sin encontrarse con las dificultades de sus colegas de Chartres:

18 No estoy de acuerdo, por lo tanto, con Wilhelm Jansen quien en su interpretación de Thierry separa categóricamente la «intellectibilitas» de la matemática: «Sie steht im Dienste der Theologen und bedarf keines Organes. Eine mira singularitas vor den übrigen Seelenkräften. An Subtilität übertrifft diese Erkenntniskraft in ihren Erwägungen die abstrakte mathematica consideratio.» (Clarambaldo de Arras, Der Kommentar des Clarenbaldus von Arras zu Boethius 'De trinitate', ed. Wilhelm Jansen, Breslau, Müller y Seifert, 1926, p. 55)

19 Para las referencias bibliográficas se remite a la nota 3. Se citará según la edición de Georg Bülow.

20 Para el uso que hacen de este verso algunos de los contemporáneos de Gundisalvo, y especialmente Pedro Abelardo, véase mi artículo, «Die Verse Römerbrief 1, $19 \mathrm{ff}$. im Verständnis Abaelards», en Patristica et Mediaevalia, 21 (2000), pp. 7688. En cuanto a Gundisalvo falta un estudio de su uso de la Sagrada Escritura que sería fundamental para comprender mejor el proceso de transmisión e integración de la cultura árabe en su obra. Klaus Reinhardt ha dado algunas ideas sobre el papel de la Sagrada Escritura en el ambiente toledano, «Bible et culture à l'époque de la reconquête de Tolède», en Jacques Huré (ed.), Tolède (1085-1985). Des traductions médiévales au mythe littéraire, París, Guy Trédaniel, 1985, pp. 135-145.

21 Domingo Gundisalvo, De processione mundi, ed. cit., p. 2, 11. 13-14.

22 Muchas han sido las voces que han acusado a Gundisalvo de ser un mediocre compilador cuyas obras no serían más que una acumulación de citas tomadas de diferentes autores, tanto árabes como latinos, y muchas veces incompatibles entre ellas. No obstante, una lectura más detenida deja ver que, como en el caso presente, Gundisalvo no sólo cita, sino que modifica a sus autores de referencia. Así también en su exposición de la clasificación boeciana de las ciencias según sus objetos, Gundisalvo invierte la definición de la matemática de «sine motu inabstracta» a «abstracta et cum motu». Véase su De divisione philosophiae, ed. cit., p. 15, 1. 8, y mi artículo mencionado en la nota 8 . 
Et rationi quidem sufficit possibilitas, demonstrationi vero necessitas, intelligentiae vero simplex et mera quaedam conceptio. Ad intelligentiam autem per intellectum sive per demonstrationem, ad intellectum per rationem, ad rationem vero per imaginationem, et ad imaginationem per sensum ascenditur. ${ }^{23}$

Aquí se tiene una relación detallada de las facultades del alma, empezando por la superior, la «intelligentia», seguida del «intellectus», luego la «ratio» y finalmente la «imaginatio» y el «sensus». Y gracias a la modificación introducida por Gundisalvo en el texto de Boecio, las tres primeras de estas facultades corresponden exactamente a los métodos de las ciencias: la inteligencia a «intelligentialiter», el intelecto a «disciplinaliter» y la razón a «rationaliter». Cambiando el «intellectualiter» de Boecio por «intelligentialiter» - otro neologismo, por cierto, que resulta de la interpretación del $D e$ trinitate - consigue establecer la misma correspondencia entre las ciencias y las facultades del alma que elaboraron Thierry y Clarembaldo sin encontrarse, en cambio, con los problemas de éstos: La física radica en la razón, la matemática en el intelecto y la ciencia divina en la inteligencia. ${ }^{24}$

Hasta aquí, la distinción de las facultades del alma podría parecer meramente nominal, pero Gundisalvo se apresura a llenar de contendio sus nuevos conceptos:

[...] ratio formas sensibliles praeter materiam, intellectus formas intelligibiles tantum, intelligentia vero unam simplicem formam utcunque, sed similiter apprehendit. ${ }^{25}$

A continuación dejará de lado las dos últimas capacidades, porque en su ensayo cosmológico le basta la razón para ascender de lo visible a Dios, al principio del cosmos:

Unde per ea, quae facta sunt, invisibilia dei intellecta creatura mundi conspicit, cum ratio ad compositionem accedit hoc modo. ${ }^{26}$

¿Pero cómo aprehende la inteligencia esta «unam simplicem formam»? ¿Y cúal es la diferencia entre el intelecto que aprehende las «formas intelligibiles» y la inteligencia que aprehende tan sólo «unam simplicem formam»? Ambos aprehenden las formas puras sin materia, ¿no se está con esto de nuevo ante el dilema con el cual se encontró Clarembaldo?

Es en su Tractatus de anima donde Gundisalvo distingue con más nitidez entre el intelecto y la inteligencia tomando de nuevo a Boecio como referencia: ${ }^{27}$

23 Doningo Gundisalvo, De processione mundi, ed. cit., p. 2, 11. 14-19.

24 La dependencia de los autores de Chartres y de Toledo, unos de los otros, es un tema discutido: Ya McKeon, Richard, «Rhetoric in the Middle Ages», en Speculum, 17 (1942), pp. 1-32, aquí p. 17, señaló semejanzas en el tratamiento de la retórica por Gundisalvo en su De divisione philosophiae y Thierry en su comentario al De inventione de Cicero. aunque no quiso pronunciarse sobre la prioridad de uno de los dos. Häring, Nikolaus, «Thierry of Chartres and Gundissalinus», en Mediaeval Studies, 26 (1964), pp. 271-286, resuelve la cuestión a favor de Gundisalvo. De otro lado, Miguel Cruz Hernández en su prólogo a Kinoshita, Noboru, op. cit., p. 19, se decanta por la prioridad de Thierry. Siendo el De processione mundi una obra tardana del arcediano de Cuéllar parece que, si existe alguna dependencia entre esta obra y el comentario de Thierry, será éste último el que influyó a Gundisalvo.

25 Domingo Gundisalvo, De processione mundi, ed. cit., p. 3, 11. 1-4.

26 Ibid., p. 3, 11. 8-10.

27 Las palabras «paucorum admodum hominum» no han podido ser localizadas en las obras de Boecio. Sin embargo, existen otros testigos que adjudican esta formulación a Boecio, por ejemplo el anónimo Liber de spiritu et anima, PL 
Sicut autem per intellectum scientia sic sapientia per intelligentiam acquiritur, quae secundum Boethium paucorum admodum hominum est et solius Dei. Intelligentia enim est altior oculus animae quo se vel Deum et aeterna contemplando speculatur. ${ }^{28}$

La inteligencia trasciende el dominio de las ciencias en sentido estricto, la física y la matemática, para ser «sapientia», sabiduría, acercándose así a Dios: Son pocos los hombres que llegan a este grado del saber que pertenece, en última instancia, solamente a Dios. Y así como el ojo corporal necesita la luz para ver, de la misma manera la inteligencia, el ojo del alma, tiene que ser iluminado para poder percibir su objeto:

Cum enim hic oculus animae qui est intelligentia in contemplationem creatoris intendit, quoniam Deus est lux, ipsa intelligentia tanta claritate divini luminis perfunditur ut ipsa intelligentia sic irradiata lux inaccessibilis tamquam forma in speculo resultare videatur: ${ }^{29}$

He aquí pues el modo del cual la inteligencia aprehende la «unam simplicem formam»: Tiene que ser iluminada por Dios, el Padre de las Luces, para que en ella como en un espejo se dé el reflejo de esta forma única y simple. La inteligencia por consecuencia no es sólo un acto del hombre, sino que en ella interviene Dios mismo: la inteligencia es divina.

Los dos pasajes que se acaban de citar tienen mucho parecido con dos formulaciones de Boecio en su Consolatio. La primera de ellas anticipa el motivo de la inteligencia como ojo que aprehende la forma simple:

Ratio vero hanc quoque transcendit speciemque ipsam quae singularibus inest universale consideratione perpendit. Intelligentiae vero celsior oculus existit; supergressa namque universitatis ambitum ipsam illam simplicem formam pura mentis acie contuetur. ${ }^{30}$

Y la segunda identifica la inteligencia con la mente divina, y incita a su lector a perseguir su unión con ella:

Si igitur uti rationis participes sumus, ita divinae iudicum mentis habere possemus, sicut imaginationem sensumque rationi cedere oportere iudicavimus, sic divinae sese menti humanam submittere rationem iustissimum censeremus. Quare in illius summae intelligentiae cacumen, si possumus, erigamur. ${ }^{31}$

Volviendo a la metodología de las ciencias y su tratamiento por Gundisalvo hay que decir pues que la «corrección» introducida con el cambio de «intellectualiter» por «intelligentialiter» no es tan

40, col. 808, así como también Pedro Abelardo en su Logica ingredientibus, ed. B. Geyer, en BGPhMa, XXI, Münster, 1919, pp. 330-331. Clarembaldo en su comentario al De trinitate de Boecio, ed. cit., p. 109, no. 8, identifica con razón esta formulación con el Timeo 51E en la traducción de Calcidio. Véase Timaeus a Calcidio translatus commentarioque instructus, ed. J. H. Waszink, Londoni et Leidae, Warburg Institute y Brill, 1962, p. 51.

28 . Domigo Gundisalvo, Tractatus de anima, ed. cit., p. 99, 11. 1-5.

29 Ibid., p. 99, 11. 22-25.

30 Boecio, Consolatio philosophiae, ed. Peip. p. 134, 11. 82-87 / Stew. p. 410, 11. 86-91. 
sólo nominal, sino que es la expresión y quizá el punto de partida de una rigurosa reestructuración de la teoría noética de Boecio. Tras su tratamiento por Gundisalvo, esta teoría, que para los autores de Chartres, como Thierry y Clarembaldo, resultaba en varios respectos equívoca o si más no problemática, se nos presenta de manera sistemática y consistente: Cada uno de los métodos de las ciencias se basa en una facultad del alma, las cuales son claramente distinguidas unas de las otras.

\section{IV. «INTELLECTUS» $Y$ «INTELLIGENTIA» EN LAS TRADUCCIONES DE GUNDISALVO}

Hasta aquí se ha expuesto el problema de la metodología boeciana y su recepción por Gundisalvo; para concluir, corresponde ahora examinar sus consecuencias en la transmisión e integración de la filosofía árabe. Ya se ha dicho que Gundisalvo tradujo junto a Avendauth unas veinte obras de pensadores arábigo-judíos al latín, gran parte de las cuales trataban de temas noéticos de una complejidad desconocida por los autores latinos..$^{32}$ Así, en los respectivos tratados noéticos de Alejandro de Afrodisia (traducido al árabe), de al-Kindi, de al-Farabi y de Avicena hay que distinguir entre una amplia gama de sentidos de la palabra 'aql, que traduce el nous griego, los cuales vienen especificados a su vez por adiciones a la misma: el intelecto en acto, el intelecto en potencia, el intelecto adquirido, el intelecto material y el intelecto agente. Mientras que los cuatro primeros de estos conceptos están ligados al alma humana, el último se concibe como separado del hombre, siendo la condición para que el intelecto posible se vuelva actual. Sin embargo, para los pensadores árabes, como por ejemplo Avicena, el intelecto agente es distinto de Dios. ${ }^{33}$ No obstante, y esto es de gran importancia para nuestro caso, todos los intelectos diferentes figuran bajo el concepto
único de 'aql.

En las traducciones latinas efectuadas por Gundisalvo, en cambio, se puede observar un desdoblamiento de este concepto como en el pasaje siguiente tomado de su traducción del Liber de anima seu sextus de naturalibus de Avicena:

[...] anima rationalis cum coniungitur formis aliquo modo coniunctionis, aptatur ut contingant in ea ex luce intelligentiae agentis ipsae formae nudae ab omni permixtione. Primum autem quod percipit de eis humanus intellectus est id quod de eis est essentiale et accidentale. ${ }^{35}$

En este pasaje el intelecto agente, al-'aql al-fa'al, se traduce por «intelligentia» destacando de esta manera su superioridad con referencia a los demás intelectos. Ahora bien, como ha demostra-

31 Ibid., ed. Peip. p. 138, 11. 44-49 / Stew. p. 418, 11. 46-52.

32 Entre estas traducciones hay que contar las siguientes: Alejandro de Afrodisia, De intellectu et intellecto, ed. Gabriel Théry, en íd., Autour du décret de 1210: II. Alexandre d'Aphrodise, Kain, Desclée, 1926, pp. 68-83; al-Kindi, De intellectu, ed. Jean Jolivet, Leiden, Brill, 1971; al-Farabi, De intellectu et intellecto, ed. Étienne Gilson, en Archives d'Histoire doctrinale et littéraire du Moyen Âge, 4 (1929), pp. 115-141; Avicena, Liber de anima seu sextus de naturalibus, 2 vols., ed. Simone van Riet, Louvain y Leiden, Peeters y Brill, 1968 y 1972.

33 Sobre la teoría de los intelectos véase por ejemplo Ramón Guerrero, Rafael, «¿Qué es filosofía en la cultura árabe?», en Jan A. Aertsen y Andreas Speer (eds.), Was ist Philosophie im Mittelalter?, en Miscellanea Mediaevalia, 26,
Berlín y New York, de Gruyter, 1998, pp. 257-270, aquí pp. 267-268. 34 Sigo a Hugonnard-Roche, Henri, «La tradition syro-arabe et la formation du vocabulaire philosophique latin», en
Jacqueline Hamesse (ed.), op. cit., pp. 59-80, aquí pp. 76-77. 
do Jean Jolivet no se trata aquí de una mera casualidad: «Cette traduction [...] utilise les mots 'intelligence' et 'intellect' d'une façon parfaitement régulière et précise. Le premier désigne toujours l'agent de la connaissance intellectuel [...] Quant au mot intellect, la traduction latine l'emploie quand il est question de ce qui est intellectuel dans l'âme humaine.» ${ }^{36}$

He aquí pues la distinción entre inteligencia y intelecto como Gundisalvo la elabora a partir del texto boeciano aplicada a Avicena. Para la recepción de la filosofía árabe esto significa que uno de sus rasgos más característicos se pierde: Mientras que ésta mantenía una clara diferencia entre el intelecto agente y Dios, con la traducción del primero por «intelligentia» Gundisalvo identifica los dos, ya que para él, como se acaba de ver, la inteligencia es divina. Esta modificación de la teoría aviceniana ha llevado al padre Étienne Gilson a hablar de un «agustinismo avicenizante» ${ }^{37}$ - término que ha sido muy discutido. ${ }^{38}$ No se pretende aquí sustituir un tópico por otro, pero si se quiere buscar un título preciso y manejable para calificar en pocas palabras la filosofía de Domingo Gundisalvo, parece mucho más apto hablar de un «boecianismo avicenizante». Con toda razón el padre Marie-Dominique Chenu ha bautizado el siglo XII como la aetas boetiana, ${ }^{39}$ la influencia de Boecio en estos tiempos es palpable por todos los sitios - y mucho más que la de San Agustín! A este respecto, Domingo Gundisalvo no representa ninguna excepción.

Como se ha apuntado al principio de este artículo, las citas de Boecio en las obras del arcediano abundan; pero su ocupación con los textos boecianos no tan sólo impregna y forma su vocabulario, sino que la confrontación con los problemas de su propia tradición, aquí la metodología de las ciencias según Boecio, exige a Gundisalvo desarrollar posiciones filosóficas propias. Son estas posiciones, en nuestro caso la rigurosa distinción entre «intelligentia» $\mathrm{y}$ «intellectus», las que a su vez intervienen en el proceso de traducción y transmisión con lo que la recepción e integración de la «nueva» y hasta entonces «desconocida» filosofía árabe viene a ser condicionada en gran parte por la tradición latino-cristiana y sus problemas inmanentes. Las consecuencias de tal condicionamiento pueden verse aún en San Tomás de Aquino quien, no sin algún desconcierto, se pronuncia de la siguiente manera sobre la diferencia entre las inteligencias y los intelectos:

In quibusdam tamen libris de arabico translatis, substantiae separatae, quas nos angelos dicimus, intelligentiae vocantur, forte propter hoc quod huiusmodi substantiae semper actu intelligunt. In libris tamen ex graeco translatis dicuntur intellectus seu mentes. ${ }^{40}$

Alexander Fidora SFB-FK 435-A2

J. W. Goethe-Universität Postfach 11 19 32. D-60054 Frankfurt am Main

35 Véase Avicena, op. cit., vol. I, p. 128, 11. 61-65.

36 Véase su artículo fundamental para todo lo expuesto, «Intellect et intelligence. Note sur la tradition arabo-latine des XIIe et XIIYe siècles», en S. Hossein Nasr (ed.), Mélanges offerts a Henry Corbin, Teherán 1977, pp. 681-702, aquí p. 689.

37 Véase Gilson, Étienne, «Les sources gréco-arabes de l'augustinisme avicennisant», en Archives d'Histoire doctrinale et littéraire de Moyen Âge, 4 (1929-1930), pp. 5-149, aquí p. 85.

38 Sobre todo por Miguel Cruz Hernández; véase por ejemplo su prólogo a Kinoshita, Noboru, op. cit., pp. 15-16.

39 Véase el capítulo VI de Chenu, Marie-Dominique, La théologie au XIle siècle, París, Vrin, 1957.

40 Tomás de Aquino, Summa theologiae, Ia, q. 79, art. 10, resp. 